\title{
Identities of the degenerate Daehee numbers with the Bernoulli numbers of the second kind arising from nonlinear differential equation
}

\author{
Sung-Soo Pyo ${ }^{\mathrm{a}}$, Taekyun Kim ${ }^{\mathrm{b}, *}$, Seog-Hoon Rim ${ }^{\mathrm{c}, *}$ \\ ${ }^{a}$ Department of Mathematics Education, Silla University, Busan, Rep. of Korea. \\ ${ }^{b}$ Department of Mathematics, Kwangwoon University, Seoul, Rep. of Korea. \\ ${ }^{c}$ Department of Mathematics Education, Kyungpook National University, Taegu, Rep. of Korea. \\ Communicated by M. Bohner
}

\begin{abstract}
In [T. Kim, D. S. Kim, H. I. Kwon, J. J. Seo, Glob. J. Pure Appl. Math., 12 (2016), 1893-1901], Kim et al. presented some identities for the Bernoulli numbers of the second kind using differential equation. Here we use this differential equation in a different way. In this paper, we deduce some identities of the degenerate Daehee numbers with the Bernoulli numbers of the second kind of order $r$. (C)2017 All rights reserved.
\end{abstract}

Keywords: Degenerate Daehee numbers, Bernoulli numbers of the second kind, nonlinear differential equation. 2010 MSC: 05A19, 11B37, 11B83, 34A34.

\section{Introduction}

For any real number $x$ and nonnegative integer $n$, throughout this paper, we denote $(x)_{n}$ for falling factorial $(x)_{n}=x(x-1)(x-2) \cdots(x-n+1)$ and $(x)_{0}=1$ and $S_{1}(m, n)$ and $S_{2}(m, n)$ denote the Stirling number of the first kind and the second kind, respectively. The $n$-th Bernoulli numbers are denoted by $B_{n}$. The definition of these numbers follows [21].

It is well-known that the $n$-th Daehee numbers of order $r$, denoted by $D_{n}^{(r)}$, are defined by the generating function

$$
\left(\frac{\log (1+t)}{t}\right)^{r}=\sum_{n=0}^{\infty} D_{n}^{(r)} \frac{t^{n}}{n !}, \quad(\text { see }[4,5,23,24]) .
$$

In the special case $r=1, D_{n}^{(1)}=D_{n}$ are called Daehee numbers.

The Daehee numbers have many interesting properties in the combinatorial special numbers. The equations shown below illustrate that there is a close relationship between Daehee numbers and Bernoulli

\footnotetext{
${ }^{*}$ Corresponding author

Email addresses: ssoopyo@gmail.com (Sung-Soo Pyo), kwangwoonmath@hanmail.net (Taekyun Kim), shrim@knu.ac.kr (Seog-Hoon Rim)

doi:10.22436/jnsa.010.12.08
}

Received 2017-09-24 
numbers:

$$
D_{n}=\sum_{m=0}^{n} B_{m} S_{1}(n, m), \quad B_{m}=\sum_{n=0}^{m} D_{n} S_{2}(m, n),
$$

where $B_{m}, S_{1}(n, m)$ and $S_{2}(n, m)$ are the $m$-th Bernouli numbers, the Stirling numbers of the first kind and the second kind, respectively (see $[1,12]$ ).

The Bernoulli numbers of the second kind of order $r([21])$ which are also called the Cauchy numbers of the first kind [19], denoted by $b_{n}^{(r)}$, are defined by the generating function

$$
\left(\frac{t}{\log (1+t)}\right)^{r}=\sum_{n=0}^{\infty} b_{n}^{(r)} \frac{t^{n}}{n !}, \quad(\text { see }[13,15,20,22]) .
$$

In [17], Kim et al. showed that the following identity holds for the Bernoulli numbers of the second kind

$$
b_{n}=\sum_{k=0}^{n} \frac{s_{1}(n, k)}{k+1} \text {. }
$$

Following Carlitz [2], several types of degenerate numbers have been presented and research has been carried out (see [3], [16]).

In [10] the degenerate Cauchy numbers of the first kind, which also are named the degenerate Bernoulli numbers of the second kind, denoted by $b_{n, \lambda}$, are introduced as follows:

$$
\frac{\log (1+\lambda t)}{\lambda \log \left(1+\frac{1}{\lambda} \log (1+\lambda t)\right)}=\sum_{n=0}^{\infty} b_{n, \lambda} \frac{t^{n}}{n !} .
$$

The degenerate Daehee numbers, denoted by $D_{n, \lambda}$, are introduced as follows (see [8]):

$$
\frac{\lambda \log \left(1+\frac{1}{\lambda} \log (1+\lambda t)\right)}{\log (1+\lambda t)}=\sum_{n=0}^{\infty} D_{n, \lambda} \frac{t^{n}}{n !} .
$$

If $\lambda$ goes to 0 , then $b_{n, \lambda}$ and $D_{n, \lambda}$ converge to $b_{n}$ and $D_{n}$, respectively.

Recently, a group of mathematicians used a differential equations to study special numbers. In [6], the Daehee numbers are considered by using differential equations arising from the generating function. New identities were obtained for degenerate Daehee numbers [8] and for Frobenius-Euler polynomials [9] using linear and nonlinear differential equations. Using differential equations, new identities for Bernoulli numbers of the second kind were obtained in [12], for degenerate Euler numbers and polynomials in [14], and for Laguerre polynomials in [18]. Jang and Kim presented some identities of the ordered Bell numbers arising from differential equation [7].

In [8], authors presented some identities of degenerate Daehee numbers arising from nonlinear differential equations. The differential equation is deduced by the function

$$
\mathrm{F}=\mathrm{F}(\mathrm{t})=\log \left(1+\frac{1}{\lambda} \log (1+\lambda \mathrm{t})\right) .
$$

So, naturally, it is valuable to study what happen if $F$ is selected as follows:

$$
F=\frac{\lambda}{\log (1+\lambda t)}
$$

In [21], Kim et al. studied the function $F=F(t)=\frac{1}{\log (1+t)}$. Some identities for the Bernoulli numbers of the second kind were deduced.

Nevertheless, in the study of (1.1), we can find some interesting identities for degenerate Daehee numbers with the Bernoulli numbers of the second kind.

In [21], Kim et al. presented some identities for the Bernoulli numbers of the second kind using differential equation. We use this differential equation in a different aspect. In this paper, we deduce some identities of the degenerate Daehee numbers with the Bernoulli numbers of the second kind. 


\section{Differential equations arising from the generating function of degenerate Daehee numbers}

In [21], Kim et al. showed that the function

$$
F=F(t)=\frac{1}{\log (1+t)}
$$

satisfies the following differential equation

$$
N ! F^{N+1}=(-1)^{N} \sum_{i=1}^{N} a_{i}(N)(1+t)^{i} F^{(i)}, \quad(N=1,2, \cdots),
$$

where $a_{1}(N)=1$ and for $2 \leqslant i \leqslant N$

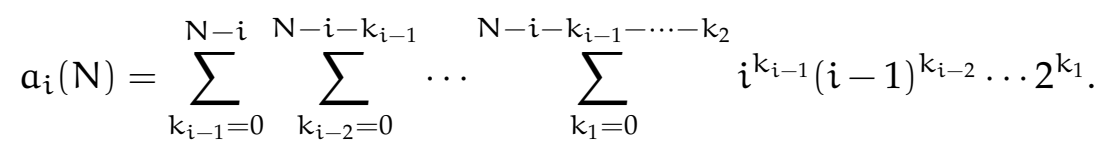

Let us consider the function

$$
F=\frac{1}{\frac{1}{\lambda} \log (1+\lambda t)}
$$

for which one can easily obtain

$$
\mathrm{F}^{2}=-(1+\lambda \mathrm{t}) \mathrm{F}^{\prime}
$$

And by taking the similar process to [21], we get

$$
N ! F^{N+1}=(-1)^{N} \sum_{i=1}^{N} a_{i}(N) \lambda^{N-i}(1+\lambda t)^{i} F^{(i)},
$$

where the coefficients $a_{i}(N)$ are the same as in (2.1).

From [21], we get

$$
\begin{aligned}
& a_{1}(N+1)=a_{1}(N), \quad a_{N+1}(N+1)=a_{N}(N), \\
& a_{i}(N+1)=i a_{i}(N)+a_{i-1}(N), \\
& a_{1}(N+1)=a_{1}(N)=\cdots=a_{1}(1)=1, \\
& a_{1}(1)=a_{2}(2)=\cdots=a_{N}(N)=1
\end{aligned}
$$

The conditions of $a_{i}(N)$ follow the Stirling numbers of the second kind, therefore we assume that (2.3) equals to

$$
\mathrm{N} ! \mathrm{F}^{\mathrm{N}+1}=(-1)^{\mathrm{N}} \sum_{\mathrm{k}=1}^{\mathrm{N}} \mathrm{S}_{2}(\mathrm{~N}, \mathrm{k}) \lambda^{\mathrm{N}-\mathrm{k}}(1+\lambda \mathrm{t})^{\mathrm{k}} \mathrm{F}^{(\mathrm{k})} .
$$

We can easily show that (2.4) is true for $N=1,2$ and this is already proved in [21]. Assume that it is true for $\mathrm{N}$. We differentiate on the both sides of (2.4),

$$
\begin{aligned}
(N+1) ! F^{N} F^{\prime}= & (-1)^{N} \sum_{k=1}^{N} S_{2}(N, k) \lambda^{N-k} \\
& \times\left\{k \lambda(1+\lambda t)^{k-1} F^{(k)}+(1+\lambda t)^{k} F^{(k+1)}\right\},
\end{aligned}
$$

and multiply by $(1+\lambda t)$ on the both sides of $(2.5)$

$$
\begin{aligned}
(N+1) ! F^{N} F^{\prime}(1+\lambda t)= & (-1)^{N} \sum_{k=1}^{N} S_{2}(N, k) \lambda^{N-k} \\
& \times\left\{k \lambda(1+\lambda t)^{k} F^{(k)}+(1+\lambda t)^{k+1} F^{(k+1)}\right\} .
\end{aligned}
$$


Applying the identity (2.2) to (2.6), we get

$$
\begin{aligned}
(N+1) ! F^{N+2}= & (-1)^{N+1} \sum_{k=1}^{N} S_{2}(N, k) \lambda^{N-k} k \lambda(1+\lambda t)^{k} F^{(k)} \\
& +(-1)^{N+1} \sum_{k=1}^{N} S_{2}(N, k) \lambda^{N-k}(1+\lambda t)^{k+1} F^{(k+1)} .
\end{aligned}
$$

Hence, we get

$$
\begin{aligned}
(N+1) ! F^{N+2}= & (-1)^{N+1} \sum_{k=2}^{N} \lambda^{N-k+1}(1+\lambda t)^{k}\left(k S_{2}(N, k)+S_{2}(N, k-1)\right) F^{(k)} \\
& +(-1)^{N+1}\left(S_{2}(N, 1) \lambda^{N}(1+\lambda t) F^{(1)}+S_{2}(N, N)(1+\lambda t)^{N+1} F^{(N+1)}\right) \\
= & (-1)^{N+1} \sum_{k=2}^{N} \lambda^{N-k+1}(1+\lambda t)^{k}\left(S_{2}(N+1, k)\right) F^{(k)} \\
& +(-1)^{N+1}\left(S_{2}(N, 1) \lambda^{N}(1+\lambda t) F^{(1)}+S_{2}(N, N)(1+\lambda t)^{N+1} F^{(N+1)}\right) .
\end{aligned}
$$

Since

$$
\begin{array}{r}
S_{2}(N, 1)=1, \quad S_{2}(N, N)=1, \quad \forall N \in \mathbb{N}, \\
S_{2}(N+1, k)=S_{2}(N, k-1)+k S_{2}(N, k),
\end{array}
$$

then (2.7) becomes

$$
(N+1) ! F^{N+2}=(-1)^{N+1} \sum_{k=1}^{N+1} \lambda^{N-k+1}(1+\lambda t)^{k} S_{2}(N+1, k) F^{(k)} .
$$

Therefore we get the following Lemma 2.1 and Corollary 2.2.

Lemma 2.1. For $\mathrm{N} \in \mathbb{N}$, the differential equation

$$
\mathrm{N} ! \mathrm{F}^{\mathrm{N}+1}=(-1)^{\mathrm{N}} \sum_{\mathrm{k}=1}^{\mathrm{N}} \mathrm{S}_{2}(\mathrm{~N}, \mathrm{k}) \lambda^{\mathrm{N}-\mathrm{k}}(1+\lambda \mathrm{t})^{\mathrm{k}} \mathrm{F}^{(\mathrm{k})},
$$

has a solution

$$
\mathrm{F}=\mathrm{F}(\mathrm{t})=\frac{\lambda}{\log (1+\lambda \mathrm{t})} .
$$

Corollary 2.2. For $\mathrm{N} \in \mathbb{N}$, the following identity holds.

$$
S_{2}(N, k)=\sum_{k_{i-1}=0}^{N-i} \sum_{k_{i-2}=0}^{N-i-k_{i-1}} \cdots \sum_{k_{1}=0}^{N-i-k_{i-1}-\cdots-k_{2}} i^{k_{i-1}}(i-1)^{k_{i-2}} \cdots 2^{k_{1}} .
$$

\section{Some identities of degenerate Daehee numbers with the degenerate Cauchy numbers of the second kind}

In [9], the degenerate Cauchy numbers which are also called the degenerate Bernoulli numbers of the second kind, denoted by $b_{n, \lambda}$, are defined by

$$
\frac{\frac{1}{\lambda} \log (1+\lambda t)}{\log \left(1+\frac{1}{\lambda} \log (1+\lambda t)\right)}=\sum_{n=0}^{\infty} b_{n, \lambda} \frac{t^{n}}{n !} .
$$


In [11], Kim introduced the degenerate Cauchy numbers of the second kind, denoted by $C_{n, \lambda}$, which are given by the generating function to be

$$
\frac{t}{\log \left(1+\frac{1}{\lambda} \log (1+\lambda t)\right)}=\sum_{n=0}^{\infty} C_{n, \lambda} \frac{t^{n}}{n !} .
$$

We represent some relations between the degenerate Bernoulli numbers of the second kind and the degenerate Daehee numbers.

According [9, Theorem 2.4], $\mathrm{C}_{0, \lambda}=1$, therefore we get

$$
\begin{aligned}
\frac{1}{\log \left(1+\frac{1}{\lambda} \log (1+\lambda t)\right)} & =\frac{1}{t}+\sum_{n=1}^{\infty} C_{n, \lambda} \frac{t^{n-1}}{n !} \\
& =\frac{1}{t}+\sum_{n=0}^{\infty} \frac{C_{n+1, \lambda}}{n+1} \frac{t^{n}}{n !} .
\end{aligned}
$$

From (3.1), we obtain

$$
\begin{aligned}
F & =\frac{\lambda}{\log (1+\lambda t)} \\
& =\frac{\lambda \log \left(1+\frac{1}{\lambda} \log (1+\lambda t)\right)}{\log (1+\lambda t)} \cdot \frac{1}{\log \left(1+\frac{1}{\lambda} \log (1+\lambda t)\right)} \\
& =\left(\sum_{r=0}^{\infty} D_{r, \lambda} \frac{t^{r}}{r !}\right)\left(\frac{1}{t}+\sum_{n=0}^{\infty} \frac{C_{n+1, \lambda}}{n+1} \frac{t^{n}}{n !}\right)
\end{aligned}
$$

For brevity, let

$$
\begin{aligned}
F_{1}(t) & =\left(\sum_{r=0}^{\infty} D_{r, \lambda} \frac{t^{r}}{r !}\right) \frac{1}{t}=\sum_{r=0}^{\infty} D_{r, \lambda} \frac{t^{r-1}}{r !} \\
& =\frac{1}{t}+\sum_{r=0}^{\infty} \frac{D_{r+1, \lambda}}{r+1} \frac{t^{r}}{r !}
\end{aligned}
$$

and

$$
\begin{aligned}
F_{2}(t) & =\left(\sum_{r=0}^{\infty} D_{r, \lambda} \frac{t^{r}}{r !}\right)\left(\sum_{n=0}^{\infty} \frac{C_{n+1, \lambda}}{n+1} \frac{t^{n}}{n !}\right) \\
& =\sum_{n=0}^{\infty} \sum_{r=0}^{n}\left(\begin{array}{l}
n \\
r
\end{array}\right) \frac{D_{n-r, \lambda} C_{r+1, \lambda}}{r+1} \frac{t^{n}}{n !} .
\end{aligned}
$$

We observe (3.5) in [21],

$$
G=\frac{1}{\log (1+t)}=\frac{t}{\log (1+t)} \frac{1}{t}=\sum_{k=0}^{\infty} \frac{b_{k+1}}{k+1} \frac{t^{k}}{k !}+\frac{1}{t} .
$$

Similar to (3.5), we obtain

$$
F=\frac{\lambda}{\log (1+\lambda t)}=\frac{\lambda t}{\log (1+\lambda t)} \frac{1}{t}=\sum_{n=0}^{\infty} \frac{b_{n+1} \lambda^{n+1}}{n+1} \frac{t^{n}}{n !}+\frac{1}{t} .
$$

From (3.2), (3.3), (3.4) and (3.6), we get the following theorem. 
Theorem 3.1. For any positive integer $n$,

$$
\frac{b_{n+1} \lambda^{n+1}}{n+1}=\frac{D_{n+1, \lambda}}{n+1}+\sum_{r=0}^{n}\left(\begin{array}{l}
n \\
r
\end{array}\right) \frac{D_{n-r, \lambda} C_{r+1, \lambda}}{r+1} .
$$

From (3.3), we get the $k$-th derivative of $F_{1}$

$$
\begin{aligned}
F_{1}^{(k)} & =\frac{(-1)^{k} k !}{t^{k+1}}+\sum_{r=k}^{\infty} \frac{D_{r+1, \lambda}(r)_{k}}{r+1} \frac{t^{r-k}}{r !} \\
& =\frac{(-1)^{k} k !}{t^{k+1}}+\sum_{r=0}^{\infty} \frac{D_{r+k+1, \lambda}}{(r+k+1)} \frac{t^{r}}{r !} \\
& =F_{11}^{(k)}+F_{12}^{(k)},
\end{aligned}
$$

where $F_{11}^{(k)}=\frac{(-1)^{k} k !}{t^{k+1}}$ and $F_{12}^{(k)}=\sum_{r=0}^{\infty} \frac{D_{r+k+1, \lambda}}{(r+k+1)} \frac{t^{r} !}{r !}$. From (3.4), also we get the $k$-th derivative of $F_{2}$

$$
\begin{aligned}
F_{2}^{(k)} & =\sum_{n=k}^{\infty} \sum_{r=0}^{n}\left(\begin{array}{c}
n \\
r
\end{array}\right) \frac{D_{n-r, \lambda} C_{r+1, \lambda}(n)_{k}}{r+1} \frac{t^{n-k}}{n !} \\
& =\sum_{n=0}^{\infty} \sum_{r=0}^{n+k}\left(\begin{array}{c}
n+k \\
r
\end{array}\right) \frac{D_{n+k-r, \lambda} C_{r+1, \lambda}}{r+1} \frac{t^{n}}{n !} .
\end{aligned}
$$

From (3.7), we have

$$
\begin{aligned}
(1+\lambda t)^{k} F_{11}^{(k)} & =\left(\sum_{j=0}^{k}(k)_{j} \lambda^{j} \frac{t^{j}}{j !}\right)\left(\frac{(-1)^{k} k !}{t^{k+1}}\right)=(-1)^{k} \sum_{j=0}^{k}(k)_{j} \lambda^{j} k ! \frac{t^{j-k-1}}{j !} \\
(1+\lambda t)^{k} F_{12}^{(k)} & =\left(\sum_{j=0}^{k}(k)_{j} \lambda^{j^{j}} \frac{j}{j !}\right)\left(\sum_{r=0}^{\infty} \frac{D_{r+k+1, \lambda}}{(r+k+1)} \frac{t^{r}}{r !}\right) \\
& =\sum_{r=0}^{\infty}\left(\sum_{j=0}^{r}\left(\begin{array}{l}
r \\
j
\end{array}\right) \frac{(k)_{r-j} \lambda^{r-j} D_{j+k+1, \lambda}}{(j+k+1)}\right) \frac{t^{r}}{r !},
\end{aligned}
$$

and

$$
\begin{aligned}
(1+\lambda t)^{k} F_{2}^{(k)} & =\left(\sum_{j=0}^{k}(k)_{j} \lambda^{j} \frac{j^{j}}{j !}\right)\left(\sum_{n=0}^{\infty} \sum_{r=0}^{n+k}\left(\begin{array}{c}
n+k \\
r
\end{array}\right) \frac{D_{n+k-r, \lambda} C_{r+1, \lambda}}{r+1} \frac{t^{n}}{n !}\right) \\
& =\sum_{n=0}^{\infty}\left(\sum_{j=0}^{n} \sum_{r=0}^{j+k}\left(\begin{array}{c}
n \\
j
\end{array}\right)\left(\begin{array}{c}
j+k \\
r
\end{array}\right) \frac{(k)_{n-j} \lambda^{n-j} D_{j+k-r, \lambda} C_{r+1, \lambda}}{r+1}\right) \frac{t^{n}}{n !} .
\end{aligned}
$$

From (3.8), we get the following equation

$$
\begin{aligned}
(-1)^{N} t^{N+1} & \sum_{k=1}^{N} S_{2}(N, k) \lambda^{N-k}(1+\lambda t)^{k} F_{11}^{(k)} \\
= & \sum_{k=1}^{N} S_{2}(N, k) \lambda^{N-k}\left(\sum_{j=0}^{k}(-1)^{N+k} k !(k)_{j} \lambda^{j} \frac{t^{j-k+N}}{j !}\right) \\
= & \sum_{j=0}^{N} \sum_{k=j}^{N}(-1)^{N+k} S_{2}(N, k) \lambda^{N-k+j} k !(k)_{j} \frac{t^{j-k+N}}{j !}
\end{aligned}
$$


By changing the variable of exponent $\mathrm{N}-\mathrm{k}+\mathrm{j}$ of $\mathrm{t}$ to $\mathrm{n}$, the right side of (3.10) becomes

$$
\begin{aligned}
& \sum_{j=0}^{N} \sum_{k=j}^{N}(-1)^{N+k} S_{2}(N, k) \lambda^{N-k+j} k !(k) j \frac{t^{j-k+N}}{j !} \\
& =\sum_{n=0}^{N}\left(\sum_{j=0}^{n}(-1)^{j-n} S_{2}(N, \alpha) \frac{\lambda^{n} \alpha !(\alpha)_{j} n !}{j !}\right) \frac{t^{n}}{n !},
\end{aligned}
$$

where $\alpha=N+j-n$.

From (3.9), we get (3.11)

$$
\begin{aligned}
t^{N+1} & \sum_{k=1}^{N} S_{2}(N, k) \lambda^{N-k}(1+\lambda t)^{k} F_{12}^{(k)} \\
& =t^{N+1} \sum_{k=1}^{N} S_{2}(N, k) \lambda^{N-k}\left(\sum_{r=0}^{\infty}\left(\sum_{j=0}^{r}\left(\begin{array}{l}
r \\
j
\end{array}\right) \frac{(k)_{r-j} \lambda^{r-j} D_{j+k+1, \lambda}}{j+k+1}\right) \frac{t^{r}}{r !}\right) \\
& =\sum_{n=0}^{\infty} \sum_{k=1}^{N} \sum_{j=0}^{n}\left(\begin{array}{l}
n \\
j
\end{array}\right) S_{2}(N, k) \lambda^{N-k} \frac{(k)_{n-j} \lambda^{n-j} D_{j+k+1, \lambda}}{j+k+1} \frac{t^{n+N+1}}{n !} \\
& =\sum_{n=N}^{\infty} \sum_{k=1}^{N} \sum_{j=0}^{\beta}\left(\begin{array}{l}
\beta \\
j
\end{array}\right) S_{2}(N, k) \frac{(k)_{\beta-j} \lambda^{n-k-1-j} D_{j+k+1, \lambda} n !}{(j+k+1) \beta !} \frac{t^{n}}{n !},
\end{aligned}
$$

where $\beta=\mathrm{n}-\mathrm{N}-1$, and

$$
\begin{aligned}
t^{N+1} & \sum_{k=1}^{N} S_{2}(N, k) \lambda^{N-k}(1+\lambda t)^{k} F_{2}^{(k)} \\
= & \sum_{k=1}^{N} S_{2}(N, k) \lambda^{N-k} \\
& \times \sum_{n=0}^{\infty}\left(\sum_{j=0}^{n} \sum_{r=0}^{n+k}\left(\begin{array}{c}
n \\
j
\end{array}\right)\left(\begin{array}{c}
j+k \\
r
\end{array}\right) \frac{(k)_{n-j} \lambda^{n-j} D_{j+k-r, \lambda} C_{r+1, \lambda}}{r+1}\right) \frac{t^{n+N+1}}{n !} \\
= & \sum_{n=N+1}^{\infty} \sum_{Y} S_{2}(N, k)\left(\begin{array}{c}
\beta \\
j
\end{array}\right)\left(\begin{array}{c}
j+k \\
r
\end{array}\right) \frac{(k)_{\beta-j} \lambda^{n-k-j-1} D_{j+k-r, \lambda} C_{r+1, \lambda} n !}{(r+1) \beta !} \frac{t^{n}}{n !},
\end{aligned}
$$

where $\sum_{Y}=\sum_{k=1}^{N} \sum_{j=0}^{n-N-1} \sum_{r=0}^{n-N-1+k}, \beta=n-N-1$.

The left hand side of (2.8) becomes

$$
\begin{aligned}
\mathrm{N} ! \mathrm{t}^{\mathrm{N}+1} \mathrm{~F}^{\mathrm{N}+1} & =\mathrm{N} !\left(\frac{\lambda t}{\log (1+\lambda \mathrm{t})}\right)^{\mathrm{N}+1} \\
& =\mathrm{N} ! \sum_{n=0}^{\infty} b_{n}^{(\mathrm{N}+1)} \frac{\lambda^{n} t^{n}}{n !} \\
& =\sum_{n=0}^{\infty} N ! b_{n}^{(N+1)} \lambda^{n} \frac{\mathrm{t}^{n}}{n !} .
\end{aligned}
$$

From (2.8), (3.11), (3.12) and (3.13), we get the following theorem. 
Theorem 3.2. For positive integer $\mathrm{N} \in \mathbb{N}$, the following holds:

- if $0 \leqslant \mathrm{n} \leqslant \mathrm{N}$, then

$$
N ! b_{m}^{(N+1)}=\left(\sum_{j=0}^{n}(-1)^{j-n} S_{2}(N, \alpha) \frac{\lambda^{n} \alpha !(\alpha)_{j} n !}{j !}\right)
$$

where $\alpha=\mathrm{N}+\mathrm{j}-\mathrm{n}$;

- if $\mathrm{N}+1 \leqslant \mathrm{n}$, then

$$
\begin{aligned}
(-1)^{N} \lambda^{n} N ! b_{m}^{(N+1)}= & \sum_{k=1}^{N} \sum_{j=0}^{\beta}\left(\begin{array}{l}
\beta \\
j
\end{array}\right) S_{2}(N, k) \frac{(k)_{\beta-j} \lambda^{n-k-1-j} D_{j+k+1, \lambda} n !}{(j+k+1) \beta !} \\
& +\sum_{Y} S_{2}(N, k)\left(\begin{array}{c}
\beta \\
j
\end{array}\right)\left(\begin{array}{c}
j+k \\
r
\end{array}\right) \frac{(k)_{\beta-j} \lambda^{n-k-j-1} D_{j+k-r, \lambda} C_{r+1, \lambda} n !}{(r+1) \beta !}
\end{aligned}
$$

where $\sum_{Y}=\sum_{k=1}^{N} \sum_{j=0}^{n-N-1} \sum_{r=0}^{n-N-1+k}, \beta=n-N-1$.

From [21, Theorem 2.2] and above Theorem 3.2, we get the following theorem.

Theorem 3.3. For $\mathrm{n} \geqslant 0, \mathrm{~N} \geqslant 1$, we have

$$
\begin{array}{r}
\lambda^{n} \sum_{i=0}^{\min \{N-1, n\}}(-1)^{i}(N-i)_{n-i}\left(\begin{array}{c}
n \\
i
\end{array}\right)\left(\begin{array}{c}
N \\
i
\end{array}\right)^{-1} S_{2}(N, N-i) \\
=\frac{1}{N !}\left(\sum_{j=0}^{n}(-1)^{j-n} S_{2}(N, \alpha) \frac{\lambda^{n} \alpha !(\alpha)_{j} n !}{j !}\right),
\end{array}
$$

where $\alpha=\mathrm{N}+\mathrm{j}-\mathrm{n}$, and

$$
\begin{aligned}
\sum_{i=0}^{N-1}(-1)^{i}(N-i)_{n-i}\left(\begin{array}{c}
n \\
i
\end{array}\right)\left(\begin{array}{c}
N \\
i
\end{array}\right)^{-1} S_{2}(N, N-i) \\
+(-1)^{N}\left(\begin{array}{c}
n \\
N+1
\end{array}\right)(N+1) \sum_{k=0}^{\beta} \sum_{i=1}^{N}\left(\begin{array}{l}
\beta \\
k
\end{array}\right)(i)_{\beta-k} S_{2}(N, i) \frac{b_{k+i+1}}{k+i+1} \\
=\frac{(-1)^{N}}{\lambda^{n} N !} \sum_{k=1}^{N} \sum_{j=0}^{\beta}\left(\begin{array}{c}
\beta \\
j
\end{array}\right) S_{2}(N, k) \frac{(k)_{\beta-j} \lambda^{n-k-1-j} D_{j+k+1, \lambda} n !}{(j+k+1) \beta !} \\
+\frac{(-1)^{N}}{\lambda^{n} N !} \sum_{Y} S_{2}(N, k)\left(\begin{array}{c}
\beta \\
j
\end{array}\right)\left(\begin{array}{c}
j+k \\
r
\end{array}\right) \frac{(k)_{\beta-j} \lambda^{n-k-j-1} D_{j+k-r, \lambda} C_{r+1, \lambda} n !}{(r+1) \beta !}
\end{aligned}
$$

where $\sum_{\mathrm{Y}}=\sum_{\mathrm{k}=1}^{\mathrm{N}} \sum_{\mathrm{j}=0}^{\mathrm{n}-\mathrm{N}-1} \sum_{\mathrm{r}=0}^{\mathrm{n}-\mathrm{N}-1+\mathrm{k}}, \mathrm{\beta}=\mathrm{n}-\mathrm{N}-1$.

\section{Additional remark}

We define higher-order sub-degenerate Daehee numbers, denoted by $d_{n, \lambda^{\prime}}^{(r)}$

$$
\left(\frac{\log \left(1+\frac{1}{\lambda} \log (1+\lambda t)\right)}{t}\right)^{r}=\sum_{n=0}^{\infty} d_{n, \lambda}^{(r)} \frac{t^{n}}{n !} .
$$


The sub-degenerate Daehee numbers make it possible to express the degenerate Daehee numbers as the second kind Bernoulli numbers. This is a kind of inversion of Theorem 3.2.

We have

$$
\begin{aligned}
\left(\frac{\lambda \log \left(1+\frac{1}{\lambda} \log (1+\lambda t)\right)}{\log (1+\lambda t)}\right)^{N+1} & =\left(\frac{\lambda t}{\log (1+\lambda t)}\right)^{N+1}\left(\frac{\log \left(1+\frac{1}{\lambda} \log (1+\lambda t)\right)}{t}\right)^{N+1} \\
& =\left(\sum_{l=0}^{\infty} \lambda^{l} b_{l}^{(N+1)} \frac{t^{l}}{l !}\right)\left(\sum_{m=0}^{\infty} d_{m}^{(N+1)} \lambda^{-m} \frac{(\log (1+\lambda t))^{m}}{m !}\right) \\
& =\left(\sum_{l=0}^{\infty} \lambda^{l} b_{l}^{(N+1)} \frac{t^{l}}{l !}\right)\left(\sum_{m=0}^{\infty} d_{m}^{(N+1)} \lambda^{-m} \sum_{k=m}^{\infty} S_{1}(k, m) \frac{\lambda^{k} t^{k}}{k !}\right) \\
& =\left(\sum_{l=0}^{\infty} \lambda^{l} b_{l}^{(N+1)} \frac{t^{l}}{l !}\right)\left(\sum_{k=0}^{\infty} \sum_{m=0}^{k} d_{m}^{(N+1)} \lambda^{k-m} S_{1}(k, m) \frac{t^{k}}{k !}\right) \\
& =\sum_{n=0}^{\infty}\left(\sum_{k=0}^{n} \sum_{m=0}^{n-k}\left(\begin{array}{l}
n \\
k
\end{array}\right) \lambda^{n} b_{n}^{(N+1)} d_{m}^{(N+1)} \lambda^{n-k-m} S_{1}(n-k, m)\right) \frac{t^{n}}{n !} .
\end{aligned}
$$

Also we get the following theorem.

Theorem 4.1. For any positive integer $n$,

$$
D_{n, \lambda}^{(N+1)}=\sum_{k=0}^{n} \sum_{m=0}^{n-k}\left(\begin{array}{l}
n \\
k
\end{array}\right) \lambda^{n} b_{n}^{(N+1)} d_{m}^{(N+1)} \lambda^{n-k-m} S_{1}(n-k, m) .
$$

\section{Acknowledgment}

We are grateful for the valuable comments of referees.

\section{References}

[1] L. Carlitz, Extended Stirling and exponential numbers, Duke Math. J., 32 (1965), 205-224. 1

[2] L. Carlitz, Degenerate Stirling, Bernoulli and Eulerian numbers, Utilitas Math., 15 (1979), 51-88. 1

[3] D. V. Dolgy, T. Kim, L.-C. Jang, H.-I. Kwon, J. J. Seo, Some identities of symmetry for the degenerate q-Bernoulli polynomials under symmetry group of degree n, Glob. J. Pure Appl. Math., 12 (2016), 4385-4394. 1

[4] B. S. El-Desouky, A. Mustafa, New results on higher-order Daehee and Bernoulli numbers and polynomials, Adv. Difference Equ., 2016 (2016), 21 pages. 1

[5] B. S. El-Desouky, A. Mustafa, F. M. Abdel-Moneim, Multiparameter Higher Order Daehee and Bernoulli Numbers and Polynomials, Applied Math., 2017 (2017), 775-785. 1

[6] G.-W. Jang, T. Kim, Revisit of identities of Daehee numbers arising from nonlinear differential equations, Proc. Jangjeon Math. Soc., 20 (2017), 163-177. 1

[7] G.-W. Jang, T. Kim, Some identities of ordered Bell numbers arising from differential equation, Adv. Stud. Contemp. Math., 27 (2017), 385-397. 1

[8] G.-W. Jang, J. Kwon, J. G. Lee, Some identities of degenerate Daehee numbers arising from nonlinear differential equation, Adv. Difference Equ., 2017 (2017), 10 pages. 1

[9] T. Kim, Identities involving Frobenius-Euler polynomials arising from non-linear differential equations, J. Number Theory, 132 (2012), 2854-2865. 1, 3

[10] T. Kim, On the degenerate Cauchy numbers and polynomials, Proc. Jangjeon Math. Soc., 18 (2015), 307-312. 1

[11] T. Kim, Degenerate Cauchy numbers and polynomials of the second kind, Adv. Stud. Contemp. Math., 27 (2017), 441449. 3

[12] D. S. Kim, T. Kim, Some identities for Bernoulli numbers of the second kind arising from a non-linear differential equation, Bull. Korean Math. Soc., 52 (2015), 2001-2010. 1

[13] T. Kim, D. S. Kim, A note on nonlinear Changhee differential equations, Russ. J. Math. Phys., 23 (2016), 88-92. 1

[14] D. S. Kim, T. Kim, A note on degenerate Eulerian numbers and polynomials, Adv. Stud. Contemp. Math., 27 (2017), 431-440. 1 
[15] D. S. Kim, T. Kim, On degenerate Bell numbers and polynomials, Rev. R. Acad. Cienc. Exactas Fis. Nat. Ser. A Math., 111 (2017), 435-446. 1

[16] D. S. Kim, T. Kim, D. V. Dolgy, A note on degenerate Bernoulli numbers and polynomials associated with p-adic invariant integral on $\mathbb{Z}_{\mathrm{p}}$, Appl. Math. Comput., 259 (2015), 198-204. 1

[17] D. S. Kim, T. Kim, G. -W. Jang, Some identities of partially degenerate Touchard polynomials arising from differential equations, Adv. Stud. Contemp. Math., 27 (2017), 243-251. 1

[18] T. Kim, D. S. Kim, K.-W. Hwang, J. J. Seo, Some identities of Laguerre polynomials arising from differential equations, Adv. Difference Equ., 2016 (2016), 9 pages. 1

[19] T. Kim, D. S. Kim, H. I. Kwon, J. J. Seo, Higher-order Cauchy Numbers and Polynomials, Appl. Math. Sci., 9 (2015), 1989-2004. 1

[20] T. Kim, D. S. Kim, H. I. Kwon, J. J. Seo, Differential equations arising from the generating function of general modified degenerate Euler numbers, Adv. Difference Equ., 2016 (2016), 7 pages. 1

[21] T. Kim, D. S. Kim, H. I. Kwon, J. J. Seo, Revisit nonlinear differential equations associated with Bernoulli numbers of the second kind, Glob. J. Pure Appl. Math., 12 (2016), 1893-1901. 1, 1, 2, 2, 2, 2, 3, 3

[22] T. Kim, D. S. Kim, T. Mansour, J. J. Seo, Linear differential equations for families of polynomials, J. Inequal. Appl., 2016 (2016), 8 pages. 1

[23] J.-W. Park, J. Kwon, A note on the Degenerate High order Daehee polynomials, Appl. Math. Sic., 9 (2015), 4635-4642. 1

[24] N. L. Wang, H. Li, Some identities on the Higher-order Daehee and Changhee Numbers, Pure Appl. Math. J., 4 (2015), 33-37. 1 